\title{
GALPROP Code for Galactic Cosmic Ray Propagation and Associated Photon Emissions
}

\author{
Igor V. Moskalenko* \\ W. W. Hansen Experimental Physics Laboratory and Kavli Institute for Particle Astrophysics and \\ Cosmology, Stanford University \\ E-mail: imosestanford.edu
}

\section{Guôlaugur Jóhannesson}

Science Institute, University of Iceland \& Nordita, KTH Royal Institute of Technology and Stockholm University

E-mail: gudlaugu@hi.is

\section{Troy A. Porter}

W. W. Hansen Experimental Physics Laboratory and Kavli Institute for Particle Astrophysics and Cosmology, Stanford University

E-mail: tporterestanford.edu

The last decade brought spectacular advances in the astrophysics of cosmic rays (CRs) and $\gamma$-ray astronomy. These observations pose a considerable challenge to conventional astrophysics thus leaving an ample discovery space for new phenomena. Understanding the conventional astrophysical backgrounds is vital in moving to the new territory. The state-of-the-art CR propagation code called GALPROP is designed to address exactly this challenge. Having 23 years of development behind it, the GALPROP code has become a de-facto standard in astrophysics of CR, diffuse $\gamma$-rays, and searches of new physics. The GALPROP code uses information from astronomy, particle, and nuclear physics to predict CRs, grays, synchrotron emission and its polarization in a self-consistent manner - it provides the modeling code unifying the many results of individual measurements in physics and astronomy spanning in energy coverage, types of instrumentation, and the nature of detected species. The range of physical validity of the GALPROP code covers sub-keV - PeV energies for particles and from micro-eV - PeV for photons. The GALPROP framework includes the code and independently developed datasets, such as interstellar gas $\left(\mathrm{H}_{2}\right.$, $\mathrm{H} \mathrm{I}, \mathrm{H} \mathrm{II}$ ), radiation and magnetic fields distributions as well as the nuclear and particle production cross sections. The code and the datasets are public and are extensively used by many experimental collaborations, and by thousands of individual researchers worldwide for interpretation of their data and for making predictions. We will present latest updates to the GALPROP framework that improve its accuracy and capabilities and will discuss its applications. As always, the latest release of the code and datasets is available through the WebRun, a service to the scientific community enabling easy use of the GALPROP via web browsers.

36th International Cosmic Ray Conference -ICRC2019-

July 24th - August 1st, 2019

Madison, WI, U.S.A.

* Speaker. 


\section{Introduction}

The last decade brought spectacular advances in the astrophysics of cosmic rays (CRs) and $\gamma$-ray astronomy. Launches of new missions employing forefront detector technologies were followed by a series of remarkable discoveries in an energy range that was already deemed well studied. Among those missions are the Payload for Antimatter Matter Exploration and Lightnuclei Astrophysics (PAMELA) launched in 2006, the Fermi Large Area Telescope (Fermi-LAT) launched in 2008, and the Alpha Magnetic Spectrometer - 02 (AMS-02) launched in 2011. Other high-expectations missions, CALorimetric Electron Telescope - CALET, and DArk Matter Particle Explorer mission - DAMPE, both launched in 2015, and Cosmic-Ray Energetics and Mass investigation - ISS-CREAM launched in 2017, are collecting data and began to deliver outstanding results ${ }^{1}$. Complementary to the Fermi-LAT, but deployed on the slopes of the Sierra Negra volcano, the High-Altitude Water Cherenkov (HAWC) $\gamma$-ray observatory was completed in 2015. It observes the sky revolving above and extends the Fermi-LAT coverage to $\sim 100 \mathrm{TeV}$. The technologies employed by these missions have enabled measurements with unmatched precision, which allow for searches of subtle signatures of new phenomena in CR and $\gamma$-ray data, e.g., the claimed precision of AMS-02 data is 1-3\%. On the other hand, built with the technology of 1970s and launched in 1977 at the dawn of the space era, Voyager 1, 2 spacecraft demonstrate unbelievable scientific longevity providing invaluable data on the CR spectra and composition at the interstellar reaches of the solar system currently at $146 \mathrm{AU}$ and $121 \mathrm{AU}$ from the Sun, correspondingly.

Besides Fermi-LAT and HAWC, indirect CR measurements are made by other multi-wavelength observatories. The International Gamma-Ray Astrophysics Laboratory (INTEGRAL), the High Energy Stereoscopic System (H.E.S.S.), Major Atmospheric Gamma-ray Imaging Cherenkov Telescopes (MAGIC), and the Very Energetic Radiation Imaging Telescope Array System (VERITAS), observe $\mathrm{keV}$ - multi-TeV emissions produced by particles in various environments. Construction of the next generation Cherenkov Telescope Array (CTA) has begun in 2017. Highresolution data in the microwave domain are provided by the Wilkinson Microwave Anisotropy Probe (WMAP) and Planck. The recent technological advances for the CR experiments, $\gamma$-ray and astronomy at other frequencies, together with the reached level of precision demonstrate that we are on the verge of major discoveries.

Meanwhile, many of these advances were made possible thanks to the solid results of earlier missions. To name only a few: ACE, SuperTIGER, ATIC, BESS, CAPRICE, CREAM, HEAO-3, HEAT, ISOMAX, TIGER, TRACER, Ulysses, etc. Earlier $\gamma$-ray missions, such COS-B, COMPTEL, and EGRET, also contributed a lot to understanding the processes of generation of diffuse emission and distribution of CRs in the Galaxy.

Understanding the origin of CRs, their acceleration mechanisms, main features of their interstellar propagation and source composition is impossible without a consistent interpretation of data from new experiments and past missions within a single framework. The tool that does exactly that is the state-of-the-art CR propagation code called GALPROP ${ }^{2}$ [1], which has about 23 years of development behind it [2,3]. All these years the GALPROP code is being actively updated and modified to address the challenges and demand of astrophysics community in its scope and

\footnotetext{
${ }^{1}$ See recent talks at NextGAPES-2019: http://www.sinp.msu.ru/contrib/NextGAPES

${ }^{2} \mathrm{http}: / /$ galprop.stanford.edu \& http://galprop.stanford.edu/webrun
} 
accuracy. Its primary goal is a reliable prediction of conventional astrophysical backgrounds, but it has options to propagate particles produced in annihilation or decay of dark matter particles, and calculate the associated electromagnetic emissions. In parallel, the most up-to-date models of the components of the interstellar medium (gas, radiation and magnetic fields) are being developed that are used by GALPROP making it a unique self-consistent model of the entire Milky Way galaxy.

This paper details the latest updates to the GALPROP code, further developments of its initially auxiliary datasets that grew into independent studies of the Galactic structure - distributions of gas, dust, radiation and magnetic fields as well as further extension of its capabilities.

\section{The GALPROP code}

The GALPROP project $[2,3]$ began in late $1996^{3}$ with two main concepts in mind. The first one is that various kinds of data, e.g., direct CR measurements, $\bar{p}, e^{ \pm}, \gamma$-rays, synchrotron radiation, and so forth, are all related to the same Galaxy and hence have to be modeled self-consistently [4]. The second is that all GALPROP-based models have to be as much realistic as possible making use of all available astronomical information, nuclear and particle data, with a minimum of simplifying assumptions [5]. The GALPROP code is available from a dedicated website ${ }^{2}$, where a 500+ core facility for users to run the code via online forms in a web-browser, WebRun, is also provided [6]. The number of registered users is currently about 1400 . Here we provide a brief description of GALPROP, for details see [1-3,6-20].

The GALPROP code solves a system of about 90 transport equations (time-dependent partial differential equations in 3D or 4D: spatial variables plus energy) with a given source distribution and boundary conditions for all $\mathrm{CR}$ species $\left({ }^{1} \mathrm{H}^{-64} \mathrm{Ni}, \bar{p}, e^{ \pm}\right)$[3]. This includes convection, distributed reacceleration, energy losses, nuclear fragmentation, radioactive decay, and production of secondary particles and isotopes. The numerical solution is based on a Crank-Nicholson implicit second-order scheme [21]. The spatial boundary conditions assume free-particle escape. For a given halo size the diffusion coefficient, as a function of momentum and propagation parameters, is determined from secondary-to-primary nuclei ratios, typically $\mathrm{B} / \mathrm{C},[\mathrm{Sc}+\mathrm{Ti}+\mathrm{V}] / \mathrm{Fe}$, and/or $\bar{p} / p$. If reacceleration is included, the momentum-space diffusion coefficient $D_{p p}$ is related to the spatial coefficient $D_{x x}\left(=\beta D_{0} R^{\delta}\right)$ [22], where $\delta=1 / 3$ for a Kolmogorov spectrum of interstellar turbulence or $\delta=1 / 2$ for an Iroshnikov-Kraichnan cascade, $R$ is the magnetic rigidity.

The GALPROP code computes a complete network of primary, secondary, and tertiary CR production starting from input source abundances. GALPROP includes K-capture, electron pick up and stripping processes, and knock-on electrons. Cross-sections are based on the extensive LANL database, nuclear codes, and parameterizations [20,23]. The most important isotopic production cross-sections are calculated using our own fits to major production channels [11,20,24]. Other cross-sections are computed using phenomenological codes $[25,26]$ renormalized to the data where exists. The nuclear reaction network is built using the Nuclear Data Sheets.

GALPROP calculates production of secondary particles in $p p-, p A-, A p-, A A$-interactions. Calculations of $\bar{p}$ production and propagation are detailed in [10,11,27]. Production of neutral mesons $\left(\pi^{0}, K^{0}, \bar{K}^{0}\right.$, etc. $)$, and secondary $e^{ \pm}$is calculated using the formalism $[28,29]$ as described

\footnotetext{
${ }^{3}$ http://sciencewatch.com/dr/erf/2009/09octerf/09octerfStronET/
} 
in [2] or recent parameterizations [30-32]. $\gamma$-ray production is calculated using the propagated CR distributions (all species), including primary $e^{-}$, secondary $e^{ \pm}$, and secondary $p$ from inelastic processes $[12,14,33]$. The inverse Compton scattering is treated using the formalism for an anisotropic photon distribution [8] with full interstellar radiation field [18,34,35]. The latter is calculated using the distributions of star classes, light scattering in the ISM and radiation transport (dust absorption and re-emission). Electron bremsstrahlung cross-section is calculated as described in [9]. Gas-related $\gamma$-ray intensities ( $\pi^{0}$-decay, bremsstrahlung) are computed from the emissivities using the column densities of molecular, atomic, and ionized hydrogen $\left(\mathrm{H}_{2}+\mathrm{H} \mathrm{I}+\mathrm{H}\right.$ II $)$ plus $10 \%$ of $\mathrm{He}$ (by number) as functions of Galactocentric radius, where the distributions of the gas components are derived from carbon monoxide $\mathrm{CO}$ and $21-\mathrm{cm} \mathrm{H}$ I surveys data. The synchrotron emission is computed using a parameterization of the Galactic magnetic field derived from observations of the pulsar rotation measures [15,36-42]. The line-of-sight integration of the corresponding emissivities with the distributions of gas, interstellar radiation and magnetic fields yields $\gamma$-ray and synchrotron sky maps. Spectra of all CR species on the chosen grid and the $\gamma$-ray and synchrotron sky maps are output in standard astronomical formats for direct comparison with data.

Similarly to conventionally produced CRs, GALPROP has well-developed options to propagate particles produced in exotic sources and processes, such as annihilation or decay of dark matter particles, or other sources as specified by the researcher, and calculate the associated emissions. It can be used alone or run in conjunction with dedicated packages, such as DarkSUSY [43]; the appropriate interface is also provided.

\section{New functionality}

The latest GALPROP code v.56 [17-19] allows arbitrarily small, even sub-parsec, grid sizes and finely sampled energy/time spans provided that it is running on a machine that has enough computing resources. Given that, the actual employed grid sizing is physically motivated and consistent with the baseline assumptions used to derive transport equations. Meanwhile, many optimizations and updates are made to enable as much realistic calculations as possible on moderately sized single-memory spaced systems with limited resources.

Besides that the latest version allows for the spatially variable diffusion coefficient, a separate injection spectrum for each isotope, scaling of the propagation parameters with the strength of the Galactic magnetic field and many other improvements including treatment of production of secondary particles and isotopes. Heliospheric propagation is treated using the Parker equation [44], where the numerical solutions are provided by the HELMoD code [45-48]. We work closely with the HELMOD team to combine their code with GALPROP to provide reliable calculations for all CR species within the heliosphere for any epoch as well as their local interstellar spectra.

Essential enhancements to the GALPROP code have been implemented to enable more efficient time-dependent CR propagation and interstellar emissions modeling. The "discrete sampler" is a new facility that produces a spatial and temporal discretized list of CR source regions from a user-supplied smooth CR spatial density distribution and time interval. This enables direct comparison of CR energy densities and interstellar emission intensity maps resulting from a steady-state and equivalent time-dependent realization from the same CR source density model. The discrete sampler uses an acceptance/rejection method with pseudo-random number generator, which allows 
full reproducibility of the discretization of the smooth density model for different luminosity evolutionary scenarios for the CR sources in the time-dependent case. This new facility has been used for recent work to investigate the effect of time/space discretized CR sources on predictions for the non-thermal interstellar emissions [49].

Among other improvements are the updated models of the spatial density distribution of the components of the interstellar gas $\left(\mathrm{H} \mathrm{II}, \mathrm{H}_{2}\right)$ [19] and of the interstellar radiation field. The spatial density distribution of the interstellar gas is a vital element in many astrophysical studies, but its determination is difficult because of the position of the observer in one location in the Galactic plane. Until recently models have employed the 2D Galactocentric azimuthally symmetric geometry approximation, but their accuracy is well behind the accuracy of available data. New 3D spatial density models for the neutral and molecular hydrogen are constructed based on empirical model fitting to gas line-survey data. The developed density models incorporate spiral arms and account for the warping of the disk, and the increasing gas scale height with radial distance from the Galactic center.

The models for the interstellar radiation field [18] are developed based on stellar and dust spatial density distributions taken from the literature that reproduce local near- to far-infrared observations. The interstellar emission models that include arms and bulges for the CR source and interstellar radiation densities provide plausible physical interpretations for features found in the residual maps from high-energy $\gamma$-ray data analysis. The 3D models for CR and interstellar radiation densities provide a more realistic basis that can be used for the interpretation of the non-thermal interstellar emissions from the Galaxy.

Finally, a couple of just-published papers provide illustrative examples of GALPROP capabilities. Model calculations of propagation of $\mathrm{CR} e^{ \pm}$and generation of the $\gamma$-ray emission in a two-zone diffusion model around the Geminga pulsar are described in [50,51]. The calculations are made for the whole Galaxy in steady state and time-dependent approximations employing a non-uniform grid for a region around Geminga and taking into account the proper motion of the pulsar. It predicts a $\gamma$-ray tail trailing the pulsar to appear because of the local over-density of electrons/positrons that is potentially detectable with Fermi-LAT. The analysis of the diffuse emission in the direction towards the outer halo of M31 (the Andromeda galaxy) is described in [52,53]. Application of the GALPROP-generated templates allows the foreground emission of the Milky Way to be removed revealing an extended $\sim 400 \mathrm{kpc}$-across $\gamma$-ray-emitting halo with the spectral shape that is dramatically different from the spectral shapes of other components of the foreground diffuse emission. Such a halo is detected for the first time that may lead to interesting conclusions.

GALPROP development is partially funded by NASA through grant NNX17AB48G.

\section{References}

[1] A. W. Strong, I. V. Moskalenko, T. A. Porter, G. Jóhannesson, E. Orlando, S. W. Digel et al., "GALPROP explanatory supplement." https://galprop.stanford.edu/download/manuals/galprop_explanatory_supplement.pdf, 2013.

[2] I. V. Moskalenko and A. W. Strong, Production and Propagation of Cosmic-Ray Positrons and Electrons, ApJ 493 (1998) 694 [astro-ph/9710124]. 
[3] A. W. Strong and I. V. Moskalenko, Propagation of Cosmic-Ray Nucleons in the Galaxy, ApJ 509 (1998) 212 [astro-ph/9807150].

[4] I. V. Moskalenko, A. W. Strong and O. Reimer, Diffuse galactic gamma rays, cosmic-ray nucleons and antiprotons, Astronomy and Astrophysics 338 (1998) L75 [arXiv: astro-ph/9808084].

[5] A. W. Strong, I. V. Moskalenko and V. S. Ptuskin, Cosmic-Ray Propagation and Interactions in the Galaxy, Annual Review of Nuclear and Particle Science 57 (2007) 285 [arXiv: astro-ph/0701517].

[6] A. E. Vladimirov, S. W. Digel, G. Jóhannesson, P. F. Michelson, I. V. Moskalenko, P. L. Nolan et al., GALPROP WebRun: An internet-based service for calculating galactic cosmic ray propagation and associated photon emissions, Computer Physics Communications 182 (2011) 1156 [1008. 3642 ].

[7] I. V. Moskalenko and E. Jourdain, Positron propagation in semi-relativistic plasmas: particle spectra and the annihilation line shape., Astronomy and Astrophysics 325 (1997) 401 [astro-ph/9702071].

[8] I. V. Moskalenko and A. W. Strong, Anisotropic Inverse Compton Scattering in the Galaxy, ApJ 528 (2000) 357 [astro-ph/9811284].

[9] A. W. Strong, I. V. Moskalenko and O. Reimer, Diffuse Continuum Gamma Rays from the Galaxy, ApJ 537 (2000) 763 [astro-ph/9811296].

[10] I. V. Moskalenko, A. W. Strong, J. F. Ormes and M. S. Potgieter, Secondary Antiprotons and Propagation of Cosmic Rays in the Galaxy and Heliosphere, ApJ 565 (2002) 280 [astro-ph/0106567].

[11] I. V. Moskalenko, A. W. Strong, S. G. Mashnik and J. F. Ormes, Challenging Cosmic-Ray Propagation with Antiprotons: Evidence for a "Fresh" Nuclei Component?, ApJ 586 (2003) 1050 [astro-ph/0210480].

[12] A. W. Strong, I. V. Moskalenko and O. Reimer, Diffuse Galactic Continuum Gamma Rays: A Model Compatible with EGRET Data and Cosmic-Ray Measurements, ApJ 613 (2004) 962 [arXiv: astro-ph/0406254].

[13] V. S. Ptuskin, I. V. Moskalenko, F. C. Jones, A. W. Strong and V. N. Zirakashvili, Dissipation of Magnetohydrodynamic Waves on Energetic Particles: Impact on Interstellar Turbulence and Cosmic-Ray Transport, ApJ 642 (2006) 902 [arXiv: astro-ph/ 0510335 ].

[14] A. W. Strong, T. A. Porter, S. W. Digel, G. Jóhannesson, P. Martin, I. V. Moskalenko et al., Global Cosmic-ray-related Luminosity and Energy Budget of the Milky Way, ApJL 722 (2010) L58 [1008.4330].

[15] A. W. Strong, E. Orlando and T. R. Jaffe, The interstellar cosmic-ray electron spectrum from synchrotron radiation and direct measurements, Astronomy and Astrophysics 534 (2011) A54 [1108.4822].

[16] A. E. Vladimirov, G. Jóhannesson, I. V. Moskalenko and T. A. Porter, Testing the Origin of High-energy Cosmic Rays, ApJ 752 (2012) 68 [1108.1023].

[17] I. V. Moskalenko, G. Jóhannesson, E. Orlando, T. A. Porter and A. W. Strong, GALPROP Code for Galactic Cosmic Ray Propagation and Associated Photon Emissions, Proc. 35th ICRC (Busan) (2017) 279. 
[18] T. A. Porter, G. Jóhannesson and I. V. Moskalenko, High-energy Gamma Rays from the Milky Way: Three-dimensional Spatial Models for the Cosmic-Ray and Radiation Field Densities in the Interstellar Medium, ApJ 846 (2017) 67 [1708.00816].

[19] G. Jóhannesson, T. A. Porter and I. V. Moskalenko, The Three-dimensional Spatial Distribution of Interstellar Gas in the Milky Way: Implications for Cosmic Rays and High-energy Gamma-ray Emissions, ApJ 856 (2018) 45 [1802.08646].

[20] Y. Génolini, D. Maurin, I. V. Moskalenko and M. Unger, Current status and desired precision of the isotopic production cross sections relevant to astrophysics of cosmic rays: $\mathrm{Li}, \mathrm{Be}, \mathrm{B}, \mathrm{C}$, and $N$, Physics Review C 98 (2018) 034611 [1803.04686].

[21] W. H. Press, S. A. Teukolsky, W. T. Vetterling and B. P. Flannery, Numerical recipes in FORTRAN. The art of scientific computing. Cambridge University Press, 2nd ed., 1992.

[22] E. S. Seo and V. S. Ptuskin, Stochastic reacceleration of cosmic rays in the interstellar medium, ApJ 431 (1994) 705.

[23] S. G. Mashnik, K. K. Gudima, I. V. Moskalenko, R. E. Prael and A. J. Sierk, CEM2K and LAQGSM codes as event generators for space-radiation-shielding and cosmic-ray-propagation applications, Advances in Space Research 34 (2004) 1288 [arXiv: nucl-th/0210065].

[24] I. V. Moskalenko and S. G. Mashnik, Evaluation of Production Cross Sections of Li, Be, B in CR, in Proc. 28th ICRC (Tsukuba), vol. 4, p. 1969, July, 2003, arXiv: astro-ph/0306367.

[25] W. R. Webber, A. Soutoul, J. C. Kish and J. M. Rockstroh, Updated Formula for Calculating Partial Cross Sections for Nuclear Reactions of Nuclei with $Z \leq 28$ and $E \geq 150 \mathrm{MeVN}$ Nucleon $^{-1}$ in Hydrogen Targets, ApJS 144 (2003) 153.

[26] R. Silberberg, C. H. Tsao and A. F. Barghouty, Updated Partial Cross Sections of Proton-Nucleus Reactions, ApJ 501 (1998) 911.

[27] M. Kachelriess, I. V. Moskalenko and S. S. Ostapchenko, New Calculation of Antiproton Production by Cosmic Ray Protons and Nuclei, ApJ 803 (2015) 54 [1502. 04158$].$

[28] C. D. Dermer, Secondary production of neutral pi-mesons and the diffuse galactic gamma radiation, Astronomy and Astrophysics 157 (1986) 223.

[29] C. D. Dermer, Binary collision rates of relativistic thermal plasmas. II - Spectra, ApJ 307 (1986) 47.

[30] T. Kamae, N. Karlsson, T. Mizuno, T. Abe and T. Koi, Parameterization of $\gamma, e^{+/-}$, and Neutrino Spectra Produced by p-p Interaction in Astronomical Environments, ApJ 647 (2006) 692.

[31] M. Kachelrieß and S. Ostapchenko, Deriving the cosmic ray spectrum from gamma-ray observations, Physics Review D 86 (2012) 043004 [1206.4705].

[32] M. Kachelriess, I. V. Moskalenko and S. S. Ostapchenko, Nuclear Enhancement of the Photon Yield in Cosmic Ray Interactions, ApJ 789 (2014) 136 [1406.0035].

[33] T. A. Porter, I. V. Moskalenko, A. W. Strong, E. Orlando and L. Bouchet, Inverse Compton Origin of the Hard X-Ray and Soft Gamma-Ray Emission from the Galactic Ridge, ApJ 682 (2008) 400 [0804.1774].

[34] I. V. Moskalenko, T. A. Porter and A. W. Strong, Attenuation of Very High Energy Gamma Rays by the Milky Way Interstellar Radiation Field, ApJL 640 (2006) L155 [arXiv: a stro-ph/0511149].

[35] T. A. Porter, I. V. Moskalenko and A. W. Strong, Inverse Compton Emission from Galactic Supernova Remnants: Effect of the Interstellar Radiation Field, ApJL 648 (2006) L29 [astro-ph/ 0607344 ]. 
[36] X. H. Sun, W. Reich, A. Waelkens and T. A. Enßlin, Radio observational constraints on Galactic 3D-emission models, Astronomy and Astrophysics 477 (2008) 573 [0711.1572].

[37] S. A. Mao, B. M. Gaensler, M. Haverkorn, E. G. Zweibel, G. J. Madsen, N. M. McClure-Griffiths et al., A Survey of Extragalactic Faraday Rotation at High Galactic Latitude: The Vertical Magnetic Field of the Milky Way Toward the Galactic Poles, ApJ 714 (2010) 1170 [1003. 4 519].

[38] T. R. Jaffe, A. J. Banday, J. P. Leahy, S. Leach and A. W. Strong, Connecting synchrotron, cosmic rays and magnetic fields in the plane of the Galaxy, MNRAS 416 (2011) 1152 [11 05.5885 ].

[39] M. S. Pshirkov, P. G. Tinyakov, P. P. Kronberg and K. J. Newton-McGee, Deriving the Global Structure of the Galactic Magnetic Field from Faraday Rotation Measures of Extragalactic Sources, ApJ 738 (2011) 192 [1103.0814].

[40] R. Jansson and G. R. Farrar, The Galactic Magnetic Field, ApJL 761 (2012) L11 [1210 . 7820 ].

[41] R. Jansson and G. R. Farrar, A New Model of the Galactic Magnetic Field, ApJ 757 (2012) 14 [1204.3662].

[42] E. Orlando and A. Strong, Galactic synchrotron emission with cosmic ray propagation models, MNRAS 436 (2013) 2127 [1309.2947].

[43] T. Bringmann, J. Edsjö, P. Gondolo, P. Ullio and L. Bergström, DarkSUSY 6: an advanced tool to compute dark matter properties numerically, JCAP 7 (2018) 033 [1802.03399].

[44] E. N. Parker, The passage of energetic charged particles through interplanetary space, Planetary and Space Science 13 (1965) 9.

[45] M. J. Boschini, S. Della Torre, M. Gervasi, D. Grandi, G. Jóhannesson, M. Kachelriess et al., Solution of Heliospheric Propagation: Unveiling the Local Interstellar Spectra of Cosmic-ray Species, ApJ 840 (2017) 115 [1704.06337].

[46] M. J. Boschini, S. Della Torre, M. Gervasi, D. Grandi, G. Jóhannesson, G. La Vacca et al., HelMod in the Works: From Direct Observations to the Local Interstellar Spectrum of Cosmic-Ray Electrons, ApJ 854 (2018) 94 [1801.04059].

[47] M. J. Boschini, S. Della Torre, M. Gervasi, D. Grandi, G. Jóhannesson, G. La Vacca et al., Deciphering the Local Interstellar Spectra of Primary Cosmic-Ray Species with HELMOD, ApJ 858 (2018) 61 [1804.06956].

[48] M. J. Boschini, S. Della Torre, M. Gervasi, G. La Vacca and P. G. Rancoita, Propagation of cosmic rays in heliosphere: The HELMOD model, Advances in Space Research 62 (2018) 2859 [1704.03733].

[49] T. A. Porter, G. Jóhannesson and I. V. Moskalenko, "High-energy gamma rays from the Milky Way: Time-dependent modeling of cosmic ray propagation and non-thermal emissions with the GALPROP code." ICRC (Madison), this conference, 2019.

[50] G. Jóhannesson, T. A. Porter and I. V. Moskalenko, Cosmic-ray propagation in light of the recent observation of geminga, ApJ 879 (2019) 91.

[51] G. Jóhannesson, T. A. Porter and I. V. Moskalenko, "Cosmic-ray propagation in light of recent HAWC observations of pulsar wind nebula.” ICRC (Madison), this conference, 2019.

[52] C. M. Karwin, S. Murgia, S. Campbell and I. V. Moskalenko, Fermi-LAT Observations of Gamma-Ray Emission Towards the Outer Halo of M31, arXiv e-prints (2019) arXiv:1903.10533 [1903.10533].

[53] C. M. Karwin, S. Murgia, S. Campbell and I. V. Moskalenko, "Fermi-LAT observations of gamma-ray emission towards the outer halo of M31.” ICRC (Madison), this conference, 2019. 